\title{
NEW EXAMPLES OF COMPACT 8-MANIFOLDS OF HOLONOMY SPIN(7)
}

\author{
Christine TAylor
}

A $\operatorname{Spin}(7)$-structure $\Phi$ on a manifold $M$ can be encoded in a 4-form given by

$$
\begin{aligned}
\Phi= & y_{1} \wedge y_{2} \wedge y_{3} \wedge y_{4}+y_{5} \wedge y_{6} \wedge y_{7} \wedge y_{8}+y_{1} \wedge y_{3} \wedge y_{5} \wedge y_{7} \\
& +y_{2} \wedge y_{4} \wedge y_{6} \wedge y_{8}-y_{1} \wedge y_{2} \wedge y_{5} \wedge y_{6}-y_{3} \wedge y_{4} \wedge y_{7} \wedge y_{8} \\
& -y_{1} \wedge y_{2} \wedge y_{7} \wedge y_{8}-y_{3} \wedge y_{4} \wedge y_{5} \wedge y_{6}-y_{1} \wedge y_{3} \wedge y_{6} \wedge y_{8} \\
& -y_{2} \wedge y_{4} \wedge y_{5} \wedge y_{7}-y_{1} \wedge y_{4} \wedge y_{5} \wedge y_{8}-y_{2} \wedge y_{3} \wedge y_{6} \wedge y_{7} \\
& -y_{1} \wedge y_{4} \wedge y_{6} \wedge y_{7}-y_{2} \wedge y_{3} \wedge y_{5} \wedge y_{8},
\end{aligned}
$$

where $\left\{y_{i}\right\}_{i=1}^{8}$ is an orthonormal basis of the tangent space $T_{m} M$. The subgroup of $G L(8, \mathbb{R})$ preserving $\Phi$ is $\operatorname{Spin}(7)$. Much analogous to the Kähler form $\omega$ on Calabi-Yau manifolds which has holonomy $\mathrm{SU}(n)$, the form $\Phi$ is parallel if and only $M$ has holonomy in $\operatorname{Spin}(7)$.

The Joyce construction of compact manifolds of holonomy Spin(7) [1] generalizes the Kummer construction of $K 3$ surfaces. Starting from $T^{4}$, let $\mathbb{Z}_{2}$ act on $T^{4}$ by negating each of the four coordinates. The resulting orbifold has 16 singular points. Each of these singular points can by resolved by gluing the so-called Eguchi-Hanson space $U$. Eguchi-Hanson space [1] is the blow-up of $\mathbb{C}^{2} /\{ \pm 1\}$ biholomorphic to $T^{*} \mathbb{C P}^{1}$. Give $\mathbb{C}^{2}$ coordinates $\left(z_{1}, z_{2}\right)$, where $d z_{1}=d x_{1}+i d x_{2}$, and $d z_{2}=d x_{3}+i d x_{4}$, and let $u=\left|z_{1}\right|^{2}+\left|z_{2}\right|^{2}$ and $t>0$. If we define

$$
f_{t}=\sqrt{u^{2}+t^{2}}+t^{2} \ln u-t^{2} \ln \left(\sqrt{u^{2}+t^{2}}+t^{2}\right),
$$

then $\omega_{t}=\frac{1}{2} i \partial \bar{\partial} f_{t}$ is the Kähler form of a Kähler metric on $U$. $\omega_{t}$ together with $\omega_{2}=d x_{1} \wedge d x_{3}-d x_{2} \wedge d x_{4}$ and $\omega_{3}=d x_{1} \wedge d x_{4}+d x_{2} \wedge d x_{3}$ form the triplet of smooth closed 2-forms on $U$ determining the Hyperkähler structure on $U$ and the Eguchi-Hanson metric with holonomy SU(2). Eguchi-Hanson metrics are asymptotic to the flat metric on $\mathbb{C}^{2} /\{ \pm 1\}$ at infinity. The orbifold resolved is $K 3$.

Moving from $T^{4}$ to $T^{8}$, and changing the group of action from $\mathbb{Z}_{2}$ to $G$, a power of $\mathbb{Z}_{2}$ 's, and resolving $T^{8}$ by gluing Eguchi-Hanson spaces to singular points, one can achieve manifolds of exceptional holonomy Spin(7).

Having obtained the resolution $\mathrm{M}$ of $T^{8} / G$ using Eguchi-Hanson spaces, it remains to be shown using analysis that there exists $\operatorname{Spin}(7)$-structure $\Phi$ on $\mathrm{M}$ with small torsion, further these structures can be deformed to one that is

Received August 30, 1999. 
torsion-free. The condition for the metric $g$ associated to $\Phi$ to have holonomy in $\operatorname{Spin}(7)$ is exactly that $\Phi$ should be torsion-free.

If $\operatorname{Hol}(g) \subset \operatorname{Spin}(7)$, one can determine $\operatorname{Hol}(g)$ by the $\hat{A}$-genus of $M$, given that $M$ is simply-connected. $\operatorname{Hol}(g)$ is $\operatorname{Spin}(7)$ iff $\hat{A}=1 ; \operatorname{SU}(4)$ iff $\hat{A}=2 ; \operatorname{Sp}(2)$ iff $\hat{A}=3$; and $\mathrm{SU}(2) \times \mathrm{SU}(2)$ iff $\hat{A}=4$.

In [1], Joyce constructed altogether 95 topologically distinct compact manifolds of $\operatorname{Spin}(7)$ holonomy; they were obtained by resolving $T^{8} / G$ where $G=\mathbb{Z}_{2}^{4}$ or $\mathbb{Z}_{2}^{5}$ and the action on $T^{8}$ preserves the $\operatorname{Spin}(7)$-structure, namely the associated form $\Phi$. In his examples, $b^{3}=16,8$, and 4 . Here we expand the collection of $\operatorname{Spin}(7)$ compact manifolds by considering $G=\mathbb{Z}_{2}^{6}$, and produce new examples of $\operatorname{Spin}(7)$ manifolds with $b^{3}=0$.

Let $\left(x_{1}, \ldots, x_{8}\right)$ be coordinates on $T^{8}=\mathbb{R}^{8} / \mathbb{Z}^{8}$, where $x_{i} \in \mathbb{R} / \mathbb{Z}$. Let $\alpha, \beta, \gamma, \delta, \epsilon$ be the involutions on $T^{8}$ defined by

$$
\begin{aligned}
& \alpha\left(\left(x_{1}, \ldots, x_{8}\right)\right)=\left(-x_{1},-x_{2},-x_{3},-x_{4}, x_{5}, x_{6}, x_{7}, x_{8}\right), \\
& \beta\left(\left(x_{1}, \ldots, x_{8}\right)\right)=\left(x_{1}, x_{2}, x_{3}, x_{4},-x_{5},-x_{6},-x_{7},-x_{8}\right), \\
& \gamma\left(\left(x_{1}, \ldots, x_{8}\right)\right)=\left(c_{1}-x_{1}, c_{2}-x_{2}, x_{3}, x_{4}, c_{5}-x_{5}, c_{6}-x_{6}, x_{7}, x_{8}\right), \\
& \delta\left(\left(x_{1}, \ldots, x_{8}\right)\right)=\left(d_{1}-x_{1}, x_{2}, d_{3}-x_{3}, x_{4}, d_{5}-x_{5}, x_{6}, d_{7}-x_{7}, x_{8}\right), \\
& \epsilon\left(\left(x_{1}, \ldots, x_{8}\right)\right)=\left(c_{1}+x_{1}, c_{2}+x_{2}, e_{3}+x_{3}, e_{4}+x_{4}, c_{5}+x_{5}, c_{6}+x_{6},\right. \\
&\left.e_{7}+x_{7}, e_{8}+x_{8}\right),
\end{aligned}
$$

where $c_{i}, d_{i}$ and $e_{i}$ take values in $\left\{0, \frac{1}{2}\right\}$. These five elements all preserve the 4 -form $\Phi$ which defines the $\operatorname{Spin}(7)$-structure, and they all commute with each other. The 95 examples found by Joyce come from the groups $G=\langle\alpha, \beta, \gamma, \delta\rangle$ and $G=\langle\alpha, \beta, \gamma, \delta, \epsilon\rangle$, where $e_{3}=e_{7}=0$, and $e_{4}=e_{8}=\frac{1}{2}$. The examples all have the property that $\sigma=b_{+}^{4}-b_{-}^{4}=64$ and $\chi=144$, where $b_{+}^{4}=103-b^{2}+b^{3}$ and $b^{3}=4,8$ or 16 . The singularities involved are of the five types specified by [1] as follows.

(i) $T^{4} \times\left(\mathbb{R}^{4} /\{ \pm 1\}\right)$, its resolution is $T^{4} \times U$, which adjusts the Betti numbers of $T^{8} / G$ by increasing $b^{2}$ by $1, b^{3}$ by $4, b_{+}^{4}$ and $b_{-}^{4}$ by 3 .

(ii) $\left(T^{4} /\{ \pm 1\}\right) \times\left(\mathbb{R}^{4} /\{ \pm 1\}\right)$, its resolution is $\left(T^{4} /\{ \pm 1\} \times U\right.$, which adjusts the Betti numbers of $T^{8} / G$ by increasing $b^{2}$ by $1, b_{+}^{4}$ and $b_{-}^{4}$ by 3 .

(iii) $\left(\mathbb{R}^{4} /\{ \pm 1\} \times\left(\mathbb{R}^{4} /\{ \pm 1\}\right.\right.$, its resolution is $U \times U$, which adjusts the Betti numbers of $T^{8} / G$ by increasing $b_{-}^{4}$ by 1 .

(iv) $T^{4} \times\left(\mathbb{R}^{4} /\{ \pm 1\}\right) / \sigma$, where $\sigma$ is an isometric involution defined by

$$
\sigma\left(\left(x_{1}, \cdots, x_{8}\right)\right)=\left(\frac{1}{2}+x_{1}, x_{2},-x_{3},-x_{4}, x_{5}, x_{6},-x_{7},-x_{8}\right),
$$

$\sigma$ acts freely on the $T^{4}$ part. There are two topologically distinct resolutions of $\left(T^{4} \times U\right) / \sigma$ due to the action of $\sigma$ on the $U$. The holomorphic resolution, where $\sigma\left(z_{1}, z_{2}\right)=\left(z_{1},-z_{2}\right)$, increases $b^{2}$ by $1, b^{3}$ by $2, b_{+}^{4}$ and $b_{-}^{4}$ by 1 ; the antiholomorphic resolution, where $\sigma\left(z_{1}, z_{2}\right)=\left(\overline{z_{1}}, \overline{z_{2}}\right)$, increases $b^{3}, b_{+}^{4}$, and $b_{-}^{4}$ all by 2 . 
(v) $\left(T^{4} /\{ \pm 1\}\right) \times\left(\mathbb{R}^{4} /\{ \pm 1\}\right) / \sigma$, where $\sigma$ is as defined above. Again, we have two topologically distinct resolutions. The holomorphic one increases $b^{2}, b_{+}^{4}$, and $b_{-}^{4}$ all by 1 ; the antiholomorphic one increases $b_{+}^{4}$ and $b_{-}^{4}$ by 2 .

Here, we will construct examples of $\operatorname{Spin}(7)$ manifolds with $b^{3}=0$.

Let $S_{g}$ denote the fixed point set of $T^{8}$ under $g \in G$. Looking at Joyce's examples closely, we see that in the examples arising from $G=\langle\alpha, \beta, \gamma, \delta\rangle, b^{3}=$ 16 , and $S_{\alpha \beta}=S_{\alpha} \cap S_{\beta}$ is a set of 256 singular points which yield 64 singularities of type (iii), $S_{\gamma}$ and $S_{\delta}$ yield singularities of type $(i)$ or $(i v)$ which increase $b^{3}$. In the examples arising from $G=\langle\alpha, \beta, \gamma, \delta, \epsilon\rangle, b^{3}=4$ or $8 ; S_{\alpha \beta}=S_{\alpha} \cap S_{\beta}$ and $S_{\alpha \beta \epsilon}=S_{\gamma} \cap S_{\alpha \beta \gamma \epsilon}$ each yield 32 singularities of type (iii), $S_{\delta}$ yield singularities of type $(i)$ or $(i v)$ which increase $b^{3}$. Furthermore, in order for the singular points to come from $S_{\alpha}, S_{\beta}, S_{\gamma}, S_{\delta}, S_{\alpha \beta}, S_{\alpha \beta \epsilon}$, and $S_{\alpha \beta \gamma \epsilon}$ only, and for them to be one of the specified 5 types and one has to require that

$$
\begin{aligned}
\left(c_{1}, c_{2}\right) & \neq(0,0), \quad\left(c_{5}, c_{6}\right) \neq(0,0), & \left(d_{1}, d_{3}\right) \neq(0,0), \\
\left(d_{5}, d_{7}\right) \neq(0,0), & \left(c_{1}, c_{5}\right) \neq\left(d_{1}, d_{5}\right), & \left(d_{3}, d_{7}\right) \neq(0,0) .
\end{aligned}
$$

When we add another generator $\eta$ to form group $G=\mathbb{Z}_{2}^{6}=\langle\alpha, \beta, \gamma, \delta, \epsilon, \eta\rangle$, so that we have 4 singular sets $S_{\alpha \beta}, S_{\alpha \beta \epsilon}, S_{\alpha \beta \eta}$, and $S_{\alpha \beta \epsilon \eta}$, each yielding 16 singularities of type (iii). Also, none of the singular sets arising from $G$ produces singularities of type $(i)$ or $(i v)$, so $b^{3}$ stays 0 .

To define $\eta$, we need to see how the singular sets $S_{\alpha \beta \eta}$ and $S_{\alpha \beta \epsilon \eta}$ can arise from singular sets which contain type $(i i)$ and $(v)$ singularities. There are three distinct possibilities ( - other possibilities are similar to one of the three).

(i) $S_{\alpha \beta \eta}=S_{\beta} \cap S_{\alpha \eta}$ and $S_{\alpha \beta \epsilon \eta}=S_{\delta} \cap S_{\alpha \beta \delta \epsilon \eta}$.

(ii) $S_{\alpha \beta \eta}=S_{\delta} \cap S_{\alpha \beta \delta \eta}$ and $S_{\alpha \beta \epsilon \eta}=S_{\beta} \cap S_{\alpha \epsilon \eta}$.

(iii) $S_{\alpha \beta \eta}=S_{\gamma} \cap S_{\alpha \beta \gamma \eta}$ and $S_{\alpha \beta \epsilon \eta}=S_{\delta} \cap S_{\alpha \beta \delta \epsilon \eta}$.

Let

$$
\begin{aligned}
\eta\left(\left(x_{1}, \ldots, x_{8}\right)\right)=\left(f_{1}+x_{1}, f_{2}+x_{2}, f_{3}+x_{3}, f_{4}+x_{4}, f_{5}+x_{5}\right. \\
\left.f_{6}+x_{6}, f_{7}+x_{7}, f_{8}+x_{8}\right)
\end{aligned}
$$

where $f_{i} \in\left\{0, \frac{1}{2}\right\}$.

In the first case, $S_{\alpha \beta \eta}=S_{\beta} \cap S_{\alpha \eta}$ implies that

$$
f_{5}=f_{6}=f_{7}=f_{8}=0,
$$

and $S_{\alpha \beta \epsilon \eta}=S_{\delta} \cap S_{\alpha \beta \delta \epsilon \eta}$ implies that

$$
c_{1}+d_{1}=f_{1}, e_{3}+d_{3}=f_{3}, c_{5}+d_{5}=f_{5}=0, d_{7}+e_{7}=f_{7}=0 .
$$

The fact that the only singularities are $S_{\alpha}, S_{\beta}, S_{\gamma}, S_{\delta}, S_{\alpha \eta}, S_{\alpha \beta \gamma \epsilon}, S_{\alpha \beta \delta \epsilon \eta}$, $S_{\alpha \beta}, S_{\alpha \beta \epsilon}, S_{\alpha \beta \eta}$, and $S_{\alpha \beta \epsilon \eta}$ imply that

$$
f_{1}=f_{2}=f_{3}=f_{4}=\frac{1}{2},
$$


and the other constants must take the following values:

$$
\begin{array}{cccccccccccc}
c_{1} & c_{2} & c_{5} & c_{6} & d_{1} & d_{3} & d_{5} & d_{7} & e_{3} & e_{4} & e_{7} & e_{8} \\
\frac{1}{2} & 0 & a & b & 0 & \frac{1}{2} & a & c & 0 & \frac{1}{2} & c & d \\
0 & \frac{1}{2} & a & b & \frac{1}{2} & 0 & a & c & \frac{1}{2} & 0 & c & d
\end{array}
$$

where $a, b, c, d \in\left\{0, \frac{1}{2}\right\}$ and $(a, b) \neq(0,0)$ and $(a, c) \neq(0,0)$.

In the second case, we can deduce from the nature of singularities that

$$
\begin{gathered}
d_{1}=f_{1}, \quad d_{3}=f_{3}, \quad d_{5}=f_{5}=c_{5}, \quad d_{7}=f_{7}=e_{7}, \quad f_{6}=c_{6}, \quad f_{8}=e_{8}, \\
c_{1}+d_{1}=f_{4}+e_{4}=e_{3}+d_{3}=c_{2}+f_{2}=\frac{1}{2}
\end{gathered}
$$

and the other constants must take the following values:

$\begin{array}{cccccccccccccc}c_{1} & c_{2} & c_{5} & c_{6} & d_{1} & d_{3} & d_{5} & d_{7} & e_{3} & e_{4} & f_{2} & f_{4} & f_{6} & f_{8} \\ 0 & \frac{1}{2} & a & b & \frac{1}{2} & 0 & a & c & \frac{1}{2} & 0 & 0 & \frac{1}{2} & b & d \\ \frac{1}{2} & 0 & a & b & 0 & \frac{1}{2} & a & c & 0 & \frac{1}{2} & \frac{1}{2} & 0 & b & d\end{array}$

where $(a, b) \neq(0,0),(a, c) \neq(0,0)$, and $(b, d) \neq(0,0)$.

In the last case, we deduce that

$$
\begin{gathered}
c_{1}=f_{2}, \quad c_{2}=f_{2}, \quad c_{5}=f_{5}, \quad c_{6}=f_{6}, \quad d_{1}=d_{5}=0, \quad d_{3}=d_{7}=\frac{1}{2}, \\
d_{3}+e_{3}=f_{3}, \quad d_{7}+e_{7}=f_{7}, \quad f_{4}+e_{4}=\frac{1}{2}, \quad f_{8}+e_{8}=\frac{1}{2},
\end{gathered}
$$

and the other constants must take the following values:

$$
\begin{array}{cccccccccccc}
c_{1} & c_{2} & c_{5} & c_{6} & e_{3} & e_{4} & e_{7} & e_{8} & f_{3} & f_{4} & f_{7} & f_{8} \\
a & b & c & d & 0 & \frac{1}{2} & \frac{1}{2} & 0 & \frac{1}{2} & 0 & 0 & \frac{1}{2} \\
a & b & c & d & \frac{1}{2} & 0 & 0 & \frac{1}{2} & 0 & \frac{1}{2} & \frac{1}{2} & 0
\end{array}
$$

where $(a, b) \neq(0,0),(c, d) \neq(0,0),(a, c) \neq(0,0)$ and $(b, d) \neq(0,0)$.

Example 1. Let $\alpha, \beta, \gamma, \delta, \epsilon$, and $\eta$ be defined as above, and let

$$
\begin{aligned}
\left(c_{1}, c_{2}, c_{5}, c_{6}\right)= & \left(\frac{1}{2}, 0,0, \frac{1}{2}\right), \quad\left(d_{1}, d_{3}, d_{5}, d_{7}\right)=\left(0, \frac{1}{2}, 0, \frac{1}{2}\right), \\
\left(e_{3}, e_{4}, e_{7}, e_{8}\right)= & \left(0, \frac{1}{2}, \frac{1}{2}, 0\right), \quad\left(f_{1}, f_{2}, f_{3}, f_{4}\right)=\left(\frac{1}{2}, \frac{1}{2}, \frac{1}{2}, \frac{1}{2}\right), \\
& \left(f_{5}, f_{6}, f_{7}, f_{8}\right)=(0,0,0,0) .
\end{aligned}
$$

We have

(i) 2 singularities of type (ii): $S_{\alpha}$ and $S_{\alpha \eta}$ each contributing 1 .

(ii) 64 singularities of type (iii): $S_{\alpha \beta}, S_{\alpha \beta \epsilon}, S_{\alpha \beta \eta}, S_{\alpha \beta \epsilon \eta}$ each contributing 16.

(iii) 12 singularities of type $(v): S_{\gamma}, S_{\delta}, S_{\alpha \beta \delta \epsilon \eta}$, and $S_{\alpha \beta \gamma \epsilon}$ each contributing 2 , and $S_{\beta}$ contributing 4 .

Computing the Betti numbers of the resolution of $T^{8} / G$, we get

$$
b^{0}=1, \quad b^{1}=0, \quad b^{2}=2+k, \quad b^{3}=0, \quad b_{+}^{4}=101-k, \quad b_{-}^{4}=37-k,
$$

where $0 \leq k \leq 12$.

In the 13 examples we find here, $\sigma=64, \chi=144$. Further,

$$
\hat{A}=\frac{1}{24}\left(-1+b^{1}-b^{2}+b_{+}^{4}-2 b_{-}^{4}\right)=1,
$$


so the resolved manifold has holonomy exactly $\operatorname{Spin}(7)$.

Example 2. Let

$$
\begin{gathered}
\left(c_{1}, c_{2}, c_{5}, c_{6}\right)=\left(\frac{1}{2}, 0, \frac{1}{2}, 0\right), \quad\left(d_{1}, d_{3}, d_{5}, d_{7}\right)=\left(0, \frac{1}{2}, \frac{1}{2}, 0\right), \\
\left(e_{3}, e_{4}, e_{7}, e_{8}\right)=\left(0, \frac{1}{2}, 0, \frac{1}{2}\right), \quad\left(f_{1}, f_{2}, f_{3}, f_{4}\right)=\left(0, \frac{1}{2}, \frac{1}{2}, 0\right), \\
\left(f_{5}, f_{6}, f_{7}, f_{8}\right)=\left(\frac{1}{2}, 0,0, \frac{1}{2}\right) .
\end{gathered}
$$

We have

(i) 1 singularity of type (ii) coming from $S_{\alpha}$.

(ii) 64 singularities of type (iii): $S_{\alpha \beta}, S_{\alpha \beta \epsilon}, S_{\alpha \beta \eta}, S_{\alpha \beta \epsilon \eta}$ each contributing 16.

(iii) 14 singularities of type $(v): S_{\gamma}, S_{\delta}, S_{\alpha \beta \delta \eta}, S_{\alpha \epsilon \eta}$, and $S_{\alpha \beta \gamma \epsilon}$ each contributing 2 , and $S_{\beta}$ contributing 4 .

We compute that

$$
b^{0}=1, \quad b^{1}=0, \quad b^{2}=1+j, \quad b^{3}=0, \quad b_{+}^{4}=102-j, \quad b_{-}^{4}=38-j,
$$

where $0 \leq j \leq 14$.

Again $\sigma=64, \chi=144$, and $\hat{A}=1$ in these 15 new examples.

As $\chi(M)=\frac{1}{8}\left(4 p_{2}-p_{1}^{2}\right), \sigma(M)=\frac{1}{45}\left(7 p_{2}-p_{1}^{2}\right)$. In the case of holonomy $\operatorname{Spin}(7), \hat{A}(M)=1=\frac{1}{45 \cdot 2^{7}}\left(7 p_{1}^{2}-4 p_{2}\right)=\frac{1}{24}\left(-1+b^{3}+\left(2 b^{2}+b^{4}\right)-3\left(b^{2}+b_{-}^{4}\right)\right)$. According to [2], the data that can be deduced physically are $b^{3}$ and $2 b^{2}+b^{4}$ up to exchange, thus also $b^{2}+b_{-}^{4}$.

Here is a table of all the important topological data associated to all the compact manifolds of holonomy $\operatorname{Spin}(7)$ found in [1] and the examples above.

\begin{tabular}{|l|l|l|l|l|}
\hline \hline $2 b^{2}+b^{4}$ & 174 & 158 & 150 & 142 \\
\hline$b^{3}$ & 16 & 8 & 4 & 0 \\
\hline$b^{2}+b_{-}^{4}$ & 55 & 47 & 43 & 39 \\
\hline$\chi$ & 144 & 144 & 144 & 144 \\
\hline$\sigma$ & 64 & 64 & 64 & 64 \\
\hline \hline
\end{tabular}

If one expands the possibilities of singularities beyond the five types specified by [1], one may obtain more examples of holonomy $\operatorname{Spin}(7)$ manifolds.

\section{References}

[1] D.D. Joyce, Compact Riemannian 8-manifolds with holonomy Spin(7), Invent. Math. 123 (1996), 507-552.

[2] S.L. Shatashvili and C. Vafa, Superstrings and Manifolds of Exceptional Holonomy, Selecta Math. (N.S.) 1 (1995), 347-381.

Harvard University, Department of Mathematics, Cambridge, MA 02138

E-mail address: ctaylor@math.harvard.edu 